\title{
Hypertext Classification Using Tensor Space Model and Rough Set Based Ensemble Classifier
}

\author{
Suman Saha, C.A. Murthy, and Sankar K. Pal \\ Center for Soft Computing Research, Indian Statistical Institute \\ \{ssaha_r, murthy, sankar\}@isical.ac.in
}

\begin{abstract}
As WWW grows at an increasing speed, a classifier targeted at hypertext has become in high demand. While document categorization is quite a mature, the issue of utilizing hypertext structure and hyperlinks has been relatively unexplored. In this paper, we introduce tensor space model for representing hypertext documents. We exploit the local-structure and neighborhood recommendation encapsulated in the proposed representation model. Instead of using the text on a page for representing features in a vector space model, we have used features on the page and neighborhood features to represent a hypertext document in a tensor space model. Tensor similarity measure is defined. We have demonstrated the use of rough set based ensemble classifier on proposed tensor space model. Experimental results of classification obtained by using our method outperform existing hypertext classification techniques.
\end{abstract}

Keywords: Hypertext classification, tensor space model, rough ensemble classifier.

\section{Introduction}

As the Web is expanding, where most Web pages are connected with hyperlinks, the role of automatic categorization of hypertext is becoming more and more important [1]. This is the case especially with the limitation of the retrieval engines; too much information to be searched and too much information retrieved. By categorizing documents a priori, the search space can be reduced dramatically and the quality of search result improved. Besides, Web users often prefer navigating through search directories as in portal sites.

In this article we have proposed a novel tensor space model for hypertext representation. Our model relies on different types of features, which are extracted from a hypertext document and its neighbors. The proposed model consists of a second order tensor for each hypertext document and a tensor component for each of the different types of features. In each tensor components a base level has been performed. The different types of classifications have been combined using rough set based ensemble classifier [2]. 


\section{Hypertext Features}

A hypertext document consists of different types of features which are found to be useful for representing a web page 3 . Written in HTML, web pages contain additional informations other than text content, such as HTML tags, hyperlinks and anchor text (Fig 1). These features can be divided into two broad classes: on-page features, which are directly located on the page to be represented, and features of neighbors, which are found on the pages related in some way with the page to be represented.

Most commonly used on-page features are URL of the web page, outgoing links of web page, HTML tags, title-headers and text body content of the web page.

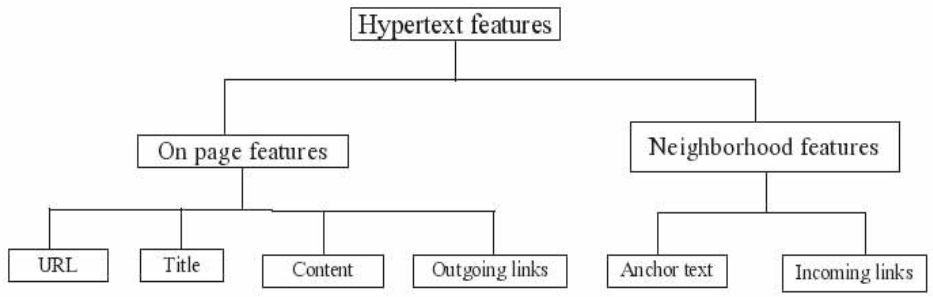

Fig. 1. Different type of features of hypertext document

\section{Proposed Method}

In this article we propose tag based tensor space model for the representation of hypertext documents and Rough Set based approach for its classification [2]. Splits of the features has been performed based on the different types of features existing in the hypertext documents. Tensor space model has been used to represent the hypertexts using the information of text content, internal mark-up structure and link structure. Classification of hypertext documents, represented as tensor, can be obtained in two ways by integrating classifier's parameters of different tensor components and by integrating classifiers output obtained on different tensor components. In this article two classification methods have been discussed using the two ways mentioned above. In the first method, a tensor similarity measure has been proposed. K-NN classification has been performed using tensor similarity measure. In the second method ensemble classification has been performed. For ensemble classification, base level classification has been performed on individual tensor components and combined classification has been obtained using rough set based ensemble classifier.

\subsection{Preprocessing and TSM Representation}

Hypertext documents are tokenized with syntactic rules and canonical forms. First we select a set of relevant features from a HTML document. For each type 
of feature an individual tensor component is constructed. A tensor component is a vector, which represents the terms of particular type corresponding to the component. The tensor space model captures the structural representation of hypertext document [4.

1) Preprocessing text content:

- The text is stemmed using Porter's stemming algorithm and stop words are removed.

- Unique words present in the text are represented as a tensor component. This tensor component corresponds to the text contents of the hypertext documents.

2) Preprocessing URL:

- A URL is first divided to yield a baseline segmentation into its components as given by the URI protocol (e.g., scheme :// host / path elements / document . extension), and further segmented wherever one or more non-alphanumeric characters appear.

- These segmented substrings are treated as words. All these words found in a URL will be represented as a tensor component corresponding to features of URLs.

3) Preprocessing anchor text:

- Anchor text is a small text content. The text is stemmed using Porter's stemming algorithm and stop words are removed.

- It is computed the same way as text content, except substituting each document by a virtual document consisting of all the anchor text inside that document.

- Unique words present in this virtual document are represented as a tensor component corresponding to features of anchor text.

4) Preprocessing title and headers:

- Title and headers are text contents. The text is stemmed using Porter's stemming algorithm and stop words are removed.

- Unique words present in these text are represented as a tensor component corresponding to features of title and headers.

5) Preprocessing in-links:

- All in-links are first divided to yield a baseline segmentation into its components as given by the URI protocol (e.g., scheme :// host / path elements / document . extension), and further segmented wherever one or more nonalphanumeric characters appear.

- The tokens obtained by segmentations of the in-links are stored in a tensor component corresponding to features of in-links. 
6) Preprocessing out-links:

- All out-links are first divided to yield a baseline segmentation into its components as given by the URI protocol and further segmented wherever one or more non-alphanumeric characters appear.

- The tokens obtained by segmentations of the out-links are stored in a tensor component corresponding to features of out-links.

\subsection{Ensemble Classifications on Tensor Space}

We now describe how we generate partitions for each one of the components of the tensor using classifiers. To generate the initial partitions for RSM we assumes a base level classifier and train it on each different tensor component. This trained classifiers provide partitions on the tensor space. Outputs of the base classifiers and the actual class information are used to construct meta level decision table. This meta data represented in the form of decision table is the input of rough set based ensemble classifier. Unlike word vector representation of web documents meta data has a simple brief format, where classifier in the ensemble contribute the existence of an attribute, values of this attribute can be any class level that is determined by the base classifier corresponding to the tensor component. So the number of attributes is the same as number of tensor components. Rough set based attribute reduction techniques eliminate superfluous attributes and create a minimal sufficient subset of attributes for a decision table. Such minimal sufficient subset of attributes, called a reduct. Once the reduct is computed we remove redundant classifiers from the ensemble and construct new reduced decision table. Rough set based decision rules extracted from this reduced decision table are applied to obtain final classification.

\section{Experimental Results}

We performed a large number of experiments to test the output of RSM. We now describe the corpora, methodology, and results.

\subsection{Data Sets}

We used four data set, Looksmart, Dmoz, webkb and Yahoo for our experiments. We crawled the Looksmart and Dmoz web directories. These directories are well known for maintaining a categorized hypertext documents. The web directories are multi-level tree-structured hierarchy. The top level of the tree, which is the first level below the root of the tree, contains 13 categories in Looksmart and 16 categories for Dmoz. Each of these categories contains sub-categories that are placed in the second level below the root. We use the top-level categories to label the web pages in our experiments. The webkb data set was collected from the WebKB project. The pages in the WebKB dataset are classified into one of the categories Student, Course, Department, Faculty, Project, Stuff and 
Other. Here there are 8077 documents in 7 categories. The largest category (Other) consists of 3025 pages; while the smallest category (Stuff) consists of only 135 pages. Another data set consists of 40000 Web pages crawled from the Yahoo topic directory (http://dir.yahoo.com). This is a big hypertext corpora, manually classified by the human experts. The extracted subset includes 33253 pages, which are distributed among 14 top level categories. The largest category (Science) consists of 4627 pages; while the smallest category (Regional) consists of only 782 pages. We processed the data sets to remove image and scripts followed by stop-words removal and stemming. Link graph has been constructed for each of the datasets for extracting neighborhood features. URLs has been segmented for extracting URL features. Finally features extracted from all the components of hypertext has been represented using tensor space model and vector space model for our experiments.

\subsection{Classification Results on TSM}

We have compared the performance of the proposed methods with existing classification techniques. The following methods are considered for comparisons.

$A$ ) Enhanced hypertext categorization using hyperlinks [5], B) Improving A Page Classifier with Anchor Extraction and Link Analysis [6], C) Fast webpage classification using URL features [7, D) Link-Local Features for Hypertext Classification [8],E) Graph based Text Classification: Learn from Your Neighbors [9], F) Web Page Classification with Heterogeneous Data Fusion [10, G) Tensor space model for hypertext representation [4] and $H$ ) The proposed technique.

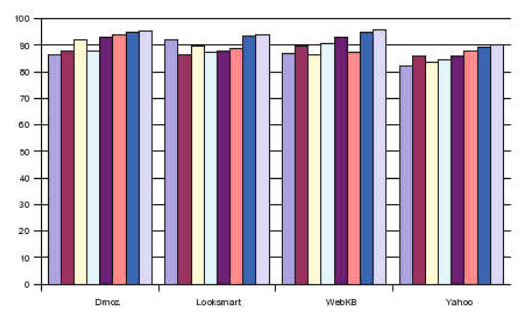

(a) Precision

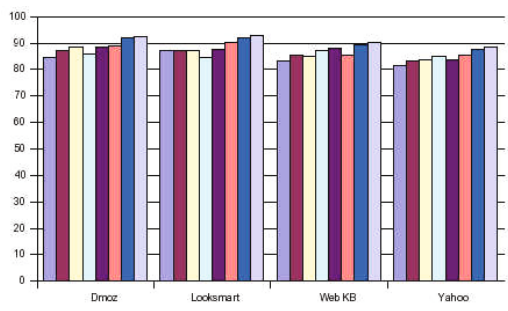

(c) Micro $F_{1}$

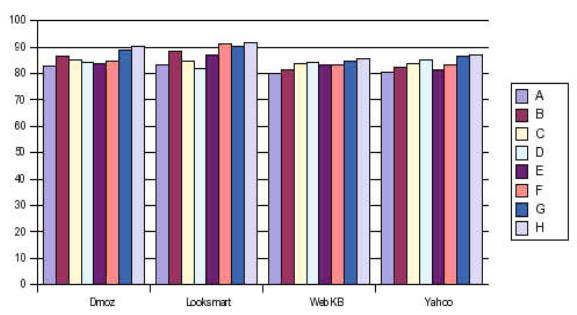

(b) Recall

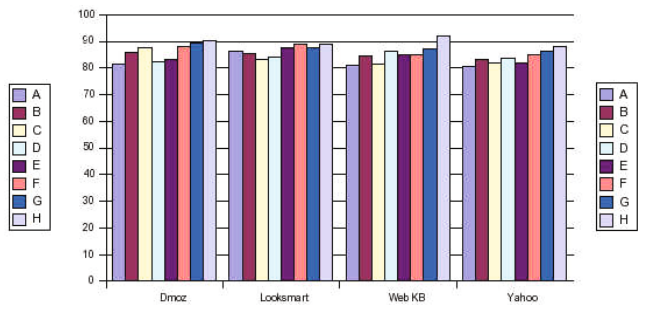

(d) Macro $F_{1}$

Fig. 2. Comparison of RSM with other hypertext classification methods 
We have compared our method with other hypertext classification algorithms. Results in terms of precision, recall, micro- $F_{1}$ and macro- $F_{1}$ of $\mathrm{A}, \mathrm{B}, \mathrm{C}, \mathrm{D}, \mathrm{E}, \mathrm{F}$, $\mathrm{G}$ and $\mathrm{H}$ have been shown in figure 2, It can be observed that performance of the proposed methods are better than others in terms of precision, recall, micro- $F_{1}$ and macro- $F_{1}$.

\section{Conclusion}

We proposed a methodology for representing hypertext documents in tensor space model. The proposed model consists of a second order tensor for each hypertext document and a tensor component for each of the different types of feature. In this representation the features extracted from URL or Title is assigned in different tensor components. Base level classification has been performed on individual tensor components. Finally combined classification has been obtained by using rough set based ensemble classifier. Two step improvement on the existing classification results of web services has been shown. In the first step we achieve better classification results by using proposed tensor space model. In the second step further improvement of the results has been obtained by using Rough set based ensemble classifier.

\section{References}

1. Yang, Y., Slattery, S., Ghani, R.: A study of approaches to hypertext categorization. Journal of Intelligent Information Systems 18(2-3), 219-241 (2002)

2. Saha, S., Murthy, C.A., Pal, S.K.: Rough set based ensemble classifier for web page classification. Fundamentae Informetica 76(1-2), 171-187 (2007)

3. Furnkranz, J.: Web mining. The Data Mining and Knowledge Discovery Handbook, pp. 899-920. Springer, Heidelberg (2005)

4. Saha, S., Murthy, C.A., Pal, S.K.: Tensor space model for hypertext representation. In: ICIT 2008: Proceedings of the 2008 International Conference on Information Technology, pp. 261-266. IEEE Computer Society, Los Alamitos (2008)

5. Chakrabarti, S., Dom, B., Indyk, P.: Enhanced hypertext categorization using hyperlinks. In: SIGMOD 1998, pp. 307-318. ACM, New York (1998)

6. Cohen, W.: Improving a page classifier with anchor extraction and link analysis (2002)

7. Kan, M.Y., Thi, H.O.N.: Fast webpage classification using url features. In: CIKM 2005: Proceedings of the 14th ACM international conference on Information and knowledge management, pp. 325-326. ACM, New York (2005)

8. Utard, H., Fürnkranz, J.: Link-local features for hypertext classification. In: Ackermann, M., Berendt, B., Grobelnik, M., Hotho, A., Mladenič, D., Semeraro, G., Spiliopoulou, M., Stumme, G., Svátek, V., van Someren, M. (eds.) EWMF 2005 and KDO 2005. LNCS (LNAI), vol. 4289, pp. 51-64. Springer, Heidelberg (2006)

9. Angelova, R., Weikum, G.: Graph-based text classification: learn from your neighbors. In: SIGIR 2006, pp. 485-492. ACM, New York (2006)

10. Xu, Z., King, I., Lyu, M.R.: Web page classification with heterogeneous data fusion. In: WWW 2007, pp. 1171-1172. ACM, New York (2007) 